\title{
Development of three level code division multiplexing over wavelength division multiplexing
}

\begin{abstract}
In this research, the Three Level Code Division Multiplexing (TLCDM) over Wavelength Division Multiplexing (WDM) is investigated in optical fiber communication system. The system is simulated using OptiSystem software over two channels at aggregated bit rate of 10 Gbps. The simulation results show that the system is able to perform BER less than 10-9 with $0 \mathrm{dBm}$ input power up to $142 \mathrm{~km}$ of optical communication system.
\end{abstract}

Keyword: Dispersion management; Optical fiber communication; TLCDM; WDM 